\title{
Plantas medicinales utilizadas por la población de la ciudad de León
}

\author{
Vanessa Elizabeth Mayorga Sánchez, Mabel Carolina Gutiérrez Cáceres y Ricardo M. Rueda Pereira*, \\ Departamento de Biología; Facultad de Ciencias; Universidad Nacional Autónoma de Nicaragua, León. (UNAN-León).
}

\section{RESUMEN}

\begin{abstract}
El propósito de este estudio es obtener información sobre las plantas medicinales que tienen mayor uso y comercialización en la población del municipio de León. El objetivo principal ha sido conocer las plantas medicinales utilizadas por la población leonesa en el periodo de juliodiciembre de 2005. Dada la falta de acceso a medicamentos esenciales con precios asequibles y la dificultad para conseguir los mismos, el uso de plantas medicinales en Nicaragua como en muchos países centroamericanos, se ha consolidado como una alternativa viable en salud, pues contribuye de forma importante en la solución de problemas relacionados a ella. De 93 barrios que se encuentran en la ciudad de León, se seleccionaron al azar veinte, cada uno con un total de veinte viviendas. Por cada vivienda seleccionada se encuestó a una persona para el levantamiento de información; resultando un total de 400 personas encuestadas. Entre las 400 encuestas, 285 (71\%) expresaron usar plantas medicinales y $115(29 \%)$ respondieron no hacer uso de este recurso. De acuerdo con los resultados obtenidos, se comprobó que las plantas medicinales representan una alternativa terapéutica para resolver los problemas de salud en la ciudad de León.
\end{abstract}

Palabras Claves: Planta medicinal, etnobotánica.

\section{INTRODUCCIÓN}

En la actualidad, se extraen en total, más de 100 plantas medicinales de los bosques; entre ellas Psychotria ipecacuanha (Raicilla), planta medicinal extraída de los bosque nicaragüenses desde 1920 en las cuencas de los ríos Mico, Siquia, Rama y San Juan. La producción nacional se exportaba por el Puerto de Bluefields a Estados Unidos y Europa. Entre 1940 y 1970, los volúmenes de exportación se mantuvieron entre 20 y 70 t por año, con un volumen máximo de 196 t en $1977{ }^{[1] .}$

Hay cinco especies que conforman más del 75 por ciento del volumen total de plantas medicinales comercializadas en el país. Estas especies son las siguientes: Smilax spp. (Zarzaparrilla), Hymenaea courbaril (Guapinol), Bursera simaruba (Jiñocuabo), Quassia amara (Hombre grande) y Petiveria alliacea (Zorrillo) ${ }^{[1]}$.

En Nicaragua los medicamentos de origen vegetal, animal y mineral, forman parte del arsenal terapéutico del país, El uso de la medicina tradicional y terapias alternativas está siendo cada vez más aceptado en países pobres y ricos. En el caso de Nicaragua, según encuestas realizadas por la OMS en el 2003 , el $60 \%$ de la población hace uso de la medicina tradicional, cuyo uso se ha incrementado debido a los bajos costos que esta representa. ${ }^{[2]}$

\footnotetext{
*Autor para correspondencia: rueda@unanleon.edu.ni
}

Cada población en el mundo posee un conocimiento heredado por sus antepasados en relación al uso de plantas medicinales, este conocimiento es una fuente importante de recursos que pueden ser utilizados por la población en general por tanto se hace necesario recopilar tal información con el propósito de documentarla y dar oportunidad a las nuevas generaciones de obtener ese conocimiento que vive en la mayoría de los casos en la memoria de las personas y se trasmite en la convivencia diaria. ${ }^{[3]}$

\section{DISEÑO METODOLÓGICO}

El municipio de León tiene una extensión territorial de $1,114 \mathrm{~km}^{2}$ y una población estimada de 194,097 habitantes. Según registro proporcionado por el MINSA, el número total de viviendas asciende a 46,333 distribuidas en el municipio.

El tipo de enfoque implementado en este trabajo de investigación ha sido descriptivo de tipo corte transversal. Recopilando datos entre el periodo de julio a diciembre del 2005, mediante encuestas etnobotánicas aplicadas a la población leonesa para su posterior organización y análisis de la misma. De los 93 barrios que se encuentran en la ciudad de León, se seleccionaron 20 al azar y 20 viviendas por cada barrio. Por cada vivienda seleccionada, se levanto una encuesta, resultando un total de 400 personas participantes. Dentro de los criterios de inclusión, las encuestas se aplicaron a personas entre 20 a 65 años, excluyendo a médicos 
y/o curanderos. La recolección de muestras botánicas se realizó en varias jornadas de campo en distintos puntos de la ciudad de León, las cuales posteriormente fueron identificadas taxonomicamente con el apoyo del Herbario Miguel Ramírez Goyena de la UNAN-León. Dentro de los materiales y equipos utilizados para documentación de la investigación se utilizaron, cámara digital, prensa botánica y estereoscopio.

\section{RESULTADOS Y DISCUSIÓN}

De un total de 400 encuestas aplicadas, 285 personas respondieron que si utilizan plantas medicinales, $242(85 \%)$ eran mujeres y $43(15 \%)$ hombres, esto seguramente debido a la ocupación predominante en el sexo femenino en atender los quehaceres del hogar.

En base al numero de personas encuestadas, las plantas medicinales de mayor frecuencia son: Plectranthus amboinicus (Orégano), Matricaria recutita (Manzanilla), Eucalyptus camaldulensis (Eucalipto), Citrus $x$ aurantium (Naranja Agria), Ambrosia peruviana (Altamiz), Mangifera indica (Mango), Cymbopogon citratos (Zacate de Limón), Citrus aurantifolia (Limón), Aloe vera (Sábila), Pluchea odorata (Salvia), Ixora coccinea (Flor de Genciana), Morinda citrifolia (Noni), Bursera graveolens (Caraña), Ocimum basilicum (Albahaca) y Persea americana (Aguacate).
La predominancia de estas plantas reportadas en las encuestas explica en parte la abundancia de esta especies como parte de los huertos caseros y jardines en León, además algunas de ellas a pesar de no ser nativas por su uso medicinal ha sido introducidas en el país y luego adoptadas por la población, tal es el caso de Eucalyptus camaldulensis, Mangifera indica. Es importante señalar que la expansión del mercado de plantas medicinales, así como el establecimiento de centros botánicos, contribuyen de manera significativa en la población para el aumento de cultivos y de ingresos en algunos casos para los hogares. Esta misma expansión del mercado influye en el incremento de la introducción de plantas no nativas que se adoptan en los hogares nicaragüenses.

El análisis de las encuestas revela que los efectos medicinales mayormente reportados para resolver sus problemas de salud se encuentran: tos, dolores menstruales, asma, problemas cardiovasculares, nervios, gripe, artritis, hipertensión, diabetes.

Adicionalmente, muchas dela plantas presentanatributos para tratamiento anti-inflamatorio, aliviar espasmo, fiebre, fortalecer defensas, combatir el insomnio, llagas y quemaduras e incluso como desinfectante.
Tabla No. 1. Usos medicinales que la población leonesa atribuye a especies de planta reportadas con fines medicinales, junio-diciembre 2005.

\begin{tabular}{|c|c|}
\hline Nombre científico & Uso medicinal reportado \\
\hline $\begin{array}{l}\text { Plectranthus amboinicus } \\
\text { (Lour.) Spreng. }\end{array}$ & Asma \\
\hline Matricaria recutita $\mathrm{L}$. & dolor menstrual y nervios \\
\hline $\begin{array}{l}\text { Eucalyptus camaldulensis } \\
\text { Dhnh. }\end{array}$ & tos, gripe, fiebre $y$ artritis \\
\hline Citrus $x$ aurantium L. & nervio, insomnio, hipertensión \\
\hline Ambrosia peruviana Willd. & corazón, dolor menstrual, nervios \\
\hline Mangifera indica $\mathrm{L}$. & anti-inflamatorio, tos, asma \\
\hline $\begin{array}{l}\text { Cymbopogon citratos (DC.) } \\
\text { Stapf }\end{array}$ & tos y gripe \\
\hline $\begin{array}{l}\text { Citrus aurantifolia (Christm.) } \\
\text { Swingle }\end{array}$ & $\begin{array}{l}\text { fiebre, anti-inflamatorio, } \\
\text { desinfectante }\end{array}$ \\
\hline Pluchea odorata (L.) Cass. & $\begin{array}{l}\text { gripe, dolor menstrual, espasmos } \\
\text { y tos }\end{array}$ \\
\hline Ixora coccinea $\mathrm{L}$. & tos \\
\hline Morinda citrifolia $\mathrm{L}$. & diabetes, fortalecer defensas, etc. \\
\hline $\begin{array}{l}\text { Bursera graveolens (Kunth) } \\
\text { Triana y Planch. }\end{array}$ & aire, dolor de oído \\
\hline Aloe vera $\mathrm{L}$. & erisipela, quemadura, llagas \\
\hline Ocimum basilicum $\mathrm{L}$. & $\begin{array}{l}\text { dolor de oído, insomnio, abrir } \\
\text { apetito }\end{array}$ \\
\hline Persea americana Mill. & dolor menstrual \\
\hline
\end{tabular}

Fotografías de algunas plantas medicinales
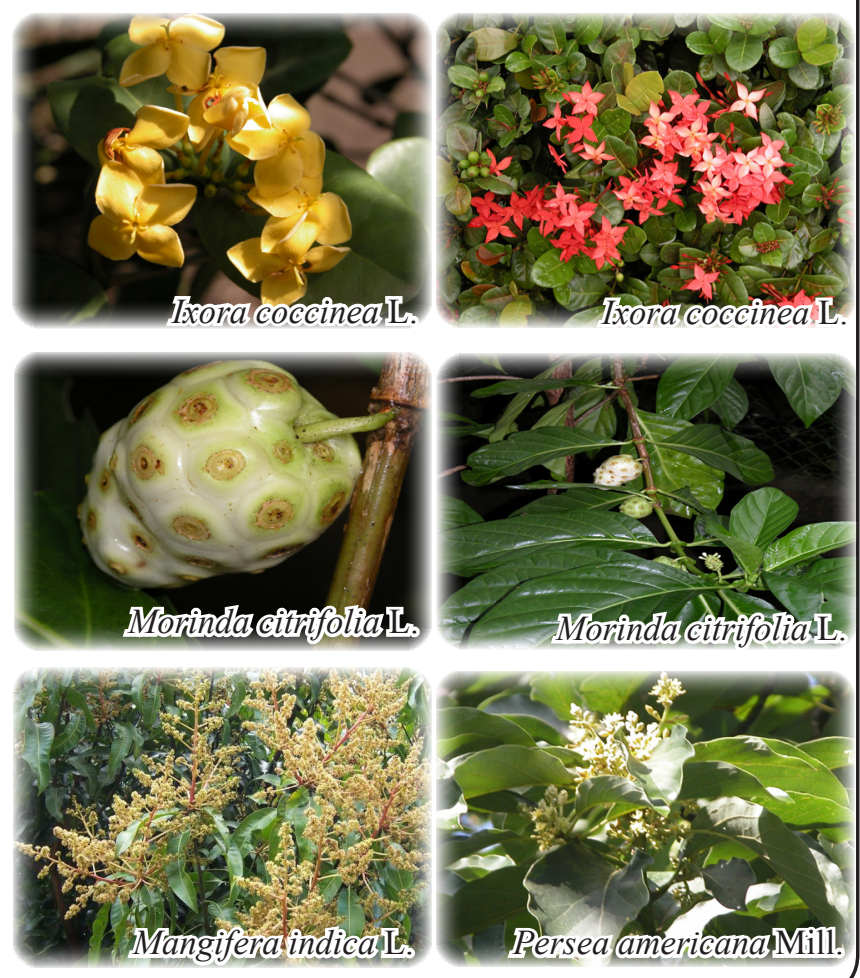


\section{CONCLUSIONES}

Las plantas mas utilizadas por la población de León según este estudio son: Plectranthus amboinicus (Orégano), Matricaria recutita (Manzanilla), Eucalyptus camaldulensis (Eucalipto), Citrus $x$ aurantium (Naranja Agria), Ambrosia peruviana (Altamiz), Mangifera indica (Mango), Cymbopogon citratos (Zacate de Limón). Los principales padecimientos que curan con las plantas medicinales son: tos, dolores menstruales, asma, insomnio, problemas cardiovasculares, nervios, gripe, artritis, hipertensión, desinfectante. Las principales fuentes de obtención de las plantas medicinales son: hogares, mercado, centros botánicos y el campo. De acuerdo con los resultados obtenidos, se comprobó que las plantas medicinales tienen importante aceptación en la población de la ciudad de León como una alternativa para resolver problemas de salud.

\section{AGRADECIMIENTOS}

Los autores agradecen a Mercedes Cáceres y Roberto Quintana por haber facilitado información para llevar a cabo la realización de la investigación y por haber revisado el manuscrito.

\section{REFERENCIAS BIBLIOGRAFICAS}

1. FAO. Evaluación de los productos forestales no maderables, Departamento de Montes, Depósitos de documentos de la FAO. Disponible en: www.fao. org.com. Acceso el 15 de octubre del (2006).

2. Guerrero, F. Campo y Agro en Nicaragua: Plantas curativas con potencial exportable. Disponible en: www.laprensa.com.ni. Acceso el 19 de octubre de (2005).

3. Tola J., Infiesta E., (2000). Las principales plantas medicinales de Europa y América. España, Ediciones Robinbook, 1ra Ed. 203 p. 